\title{
Fixing Numbers of Graphs and Groups
}

\author{
Courtney R. Gibbons \\ University of Nebraska - Lincoln \\ Department of Mathematics \\ 228 Avery Hall \\ PO Box 880130 \\ Lincoln, NE 68588-0130 \\ s-cgibbon5@math.unl.edu
}

\author{
Joshua D. Laison \\ Mathematics Department \\ Willamette University \\ 900 State St. \\ Salem, OR 97301 \\ jlaison@willamette.edu
}

Submitted: Sep 11, 2006; Accepted: Mar 12, 2009; Published: Mar 20, 2009

Mathematics Subject Classification: 05C25

\begin{abstract}
The fixing number of a graph $G$ is the smallest cardinality of a set of vertices $S$ such that only the trivial automorphism of $G$ fixes every vertex in $S$. The fixing set of a group $\Gamma$ is the set of all fixing numbers of finite graphs with automorphism group $\Gamma$. Several authors have studied the distinguishing number of a graph, the smallest number of labels needed to label $G$ so that the automorphism group of the labeled graph is trivial. The fixing number can be thought of as a variation of the distinguishing number in which every label may be used only once, and not every vertex need be labeled. We characterize the fixing sets of finite abelian groups, and investigate the fixing sets of symmetric groups.
\end{abstract}

\section{Introduction}

In this paper we investigate breaking the symmetries of a finite graph $G$ by labeling its vertices. There are two standard techniques to do this. The first is to label all of the vertices of $G$ with $k$ distinct labels. A labeling is distinguishing if no nontrivial automorphism of $G$ preserves the vertex labels. The distinguishing number of $G$ is the minimum number of labels used in any distinguishing labeling $[1,13]$. The distinguishing chromatic number of $G$ is the minimum number of labels used in any distinguishing labeling which is also a proper coloring of $G$ [6].

The second technique is to label a subset of $k$ vertices of $G$ with $k$ distinct labels. The remaining labels can be thought of as having the null label. We say that a labeling of $G$ is fixing if no non-trivial automorphism of $G$ preserves the vertex labels, and the fixing number of $G$ is the minimum number of labels used in any fixing labeling. 


\section{Fixing Graphs}

More formally, suppose that $G$ is a finite graph and $v$ is a vertex of $G$. The stabilizer of $v, \operatorname{stab}(v)$, is the set of group elements $\{g \in \operatorname{Aut}(G) \mid g(v)=v\}$. The (vertex) stabilizer of a set of vertices $S \subseteq V(G)$ is $\operatorname{stab}(S)=\{g \in \operatorname{Aut}(G) \mid g(v)=v$ for all $v \in S\}$. A vertex $v$ is fixed by a group element $g \in \operatorname{Aut}(G)$ if $g \in \operatorname{stab}(v)$. A set of vertices $S \subseteq V(G)$ is a fixing set of $G$ if $\operatorname{stab}(S)$ is trivial. In this case we say that $S$ fixes $G$. The fixing number fix $(G)$ of a graph $G$ is the smallest cardinality of a fixing set of $G[3,5,9]$.

Equivalently, $S$ is a fixing set of the graph $G$ if whenever $g \in \operatorname{Aut}(G)$ fixes every vertex in $S, g$ is the identity automorphism. A set of vertices $S$ is a determining set of $G$ if whenever two automorphisms $g, h \in \operatorname{Aut}(G)$ agree on $S$, then they agree on $G$, i.e., they are the same automorphism [3]. The following lemma shows that these two definitions are equivalent.

Lemma 1. A set of vertices is a fixing set if and only if it is a determining set.

Proof. Suppose that $S$ is a determining set. Since the identity automorphism $e$ fixes every vertex in $S$, then by the definition of a determining set, every other element $g \in \operatorname{Aut}(G)$ that fixes every vertex in $S$ must be the identity. Therefore $S$ is a fixing set. Conversely, suppose that $S$ is a fixing set. Let $g$ and $h$ agree on $S$. Then $g^{-1} h$ must fix every element in $S$. Hence by the definition of a fixing set, $g^{-1} h=e$, so $g=h$. Therefore $S$ is a determining set.

Suppose $G$ is a graph with $n$ vertices. Since fixing all but one vertex of $G$ necessarily fixes the remaining vertex, we must have $\operatorname{fix}(G) \leq n-1$. In fact, suppose that any $n-2$ vertices have been fixed in $G$, yet $G$ still has a non-trivial automorphism. Then this automorphism must be the transposition of the remaining two vertices. This implies that the only graphs which have $\operatorname{fix}(G)=n-1$ are the complete graphs and the empty graphs. On the other hand, the graphs with $\operatorname{fix}(G)=0$ are the rigid graphs [1], which have trivial automorphism group. In fact, almost all graphs are rigid [2], so most graphs have fixing number 0 .

The $\boldsymbol{o r b i t}$ of a vertex $v$, orb $(v)$, is defined to be the set of vertices $\{w \in V(G) \mid g(v)=$ $w$ for some $g \in \operatorname{Aut}(G)\}$. The Orbit-Stabilizer Theorem says that for any vertex $v$ in $G$, $|\operatorname{Aut}(G)|=|\operatorname{stab}(v)||\operatorname{orb}(v)|[12]$. So when we are building a minimal fixing set of $G$, heuristically it makes sense to choose vertices with orbits as large as possible. This leads us to consider the following algorithm for determining the fixing number of a finite graph $G$ :

\section{The Greedy Fixing Algorithm.}

1. Find a vertex $v \in G$ with $|\operatorname{stab}(v)|$ as small as possible (equivalently, with $|\operatorname{orb}(v)|$ as large as possible).

2. Fix $v$ and repeat.

3. Stop when the stabilizer of the fixed vertices is trivial. 
The set of vertices fixed by the greedy fixing algorithm must be a fixing set. We define the greedy fixing number fix greedy $(G)$ of the graph $G$ to be the number of vertices fixed by the greedy fixing algorithm.

Open Question. Is $\operatorname{fix}_{\text {greedy }}(G)$ well-defined for every finite graph $G$ ? In other words, is there a finite graph for which two different choices in Step 1 of the greedy fixing algorithm produce two different fixing sets of different sizes?

If $\operatorname{fix}_{\text {greedy }}(G)$ is well-defined, we must have $\operatorname{fix}(G) \leq \operatorname{fix}_{\text {greedy }}(G)$. We use this same technique to derive upper bounds on the fixing sets of groups in the next section.

Open Question. Assuming $\operatorname{fix}_{\text {greedy }}(G)$ is well-defined, is there a graph $G$ for which $\operatorname{fix}(G) \neq \operatorname{fix}_{\text {greedy }}(G)$ ?

\section{$3 \quad$ Fixing Sets of Groups}

Following Albertson and Collins' exposition of distinguishing sets of groups [1], we define the fixing set of a finite group $\Gamma$ to be $\operatorname{fix}(\Gamma)=\{\operatorname{fix}(G) \mid G$ is a finite graph with $\operatorname{Aut}(G) \cong$ $\Gamma\}$. Our goal for the remainder of the paper is to find the fixing sets of a few well-known finite groups. We begin by describing two procedures that can be used to generate specific examples.

For every graph $G$, the natural representation of the elements of $\operatorname{Aut}(G)$ as permutations of the vertices of $G$ is a group action of the group $\operatorname{Aut}(G)$ on the set $V(G)$. Furthermore, $\operatorname{Aut}(G)$ acts faithfully on $G$, i.e., the only element of $\operatorname{Aut}(G)$ that fixes every vertex in $G$ is the identity element. A group action of $\Gamma$ on a graph $G$ is vertextransitive if, given any two vertices $u, v \in V(G)$, there is an element of $\Gamma$ that sends $u$ to $v$. The following theorem appears in [7].

Theorem 2. Let $\Gamma$ be a finite group. The set of vertex-transitive actions of $\Gamma$ on all possible sets of vertices $V$ is in one-to-one correspondence with the conjugacy classes of subgroups of $\Gamma$. Specifically, if $v$ is any vertex in $V$, the action of $\Gamma$ on $V$ is determined by the conjugacy class of $\operatorname{stab}(v)$.

Suppose that $\Gamma$ is the automorphism group of a graph $G$. Then $\Gamma$ acts transitively on each orbit of the vertices of $G$ under $\Gamma$. Hence given a group $\Gamma$, to find a graph $G$ with automorphism group $\Gamma$, we choose a set of subgroups of $\Gamma$ and generate the orbits of vertices of $G$ corresponding to these subgroups using Theorem 2. There are two aspects of this construction which make the procedure difficult. First, the action of $\Gamma$ on the entire graph $G$ must be faithful for $\Gamma$ to be a valid automorphism group. Second, after we construct orbits of vertices, we must construct the edges of $G$ so that the set of permutations of vertices in $\Gamma$ is exactly the set of edge-preserving permutations of $G$. However, this is not always possible.

An alternative approach uses the Orbit-Stabilizer Theorem. Given a graph $G$ and a fixing set $S$ of $G$, we order the elements of $S$ as, say, $v_{1}, \ldots, v_{k}$, and we consider the chain of $\operatorname{subgroups} e=\operatorname{stab}\left(\left\{v_{1}, \ldots, v_{k}\right\}\right) \leq \operatorname{stab}\left(\left\{v_{1}, \ldots, v_{k-1}\right\}\right) \leq \ldots \leq \operatorname{stab}\left(v_{1}\right) \leq \operatorname{Aut}(G)$. 
If $o\left(v_{i}\right)$ is the number of vertices in $\operatorname{orb}\left(v_{i}\right)$ under the action of $\operatorname{stab}\left(\left\{v_{1}, \ldots, v_{i-1}\right\}\right)$, then $\left|\operatorname{stab}\left(\left\{v_{1}, \ldots, v_{i-1}\right\}\right)\right|=o\left(v_{i}\right)\left|\operatorname{stab}\left(\left\{v_{1}, \ldots, v_{i}\right\}\right)\right|$. So $|\operatorname{Aut}(G)|=\Pi_{1 \leq i \leq k} O\left(v_{i}\right)$. Hence given a finite group $\Gamma$, to find a graph $G$ with automorphism group $\Gamma$ and fixing number $k$, we choose a sequence of orbit sizes $\left(o\left(v_{1}\right), \ldots, o\left(v_{k}\right)\right)$ whose product is $|\Gamma|$ and look for a graph with these orbit sizes. Both of these procedures were used to generate examples given below.

We now prove a few theorems valid for the fixing set of any finite group. Let $\Gamma$ be a group generated by the set of elements $\mathcal{G}=\left\{g_{1}, g_{2}, \ldots g_{k}\right\}$. The Cayley graph $C(\Gamma, \mathcal{G})$ of $\Gamma$ with respect to the generating set $\mathcal{G}$ is a directed, edge-labeled multigraph with a vertex for each element of $\Gamma$, and a directed edge from the group element $h_{1}$ to the group element $h_{2}$ labeled with the generator $g \in \mathcal{G}$ if and only if $g h_{1}=h_{2}$.

We obtain an undirected, edge-unlabeled graph $F(\Gamma, \mathcal{G})$ from the Cayley graph $C(\Gamma, \mathcal{G})$ by replacing each directed, labeled edge of $C(\Gamma, \mathcal{G})$ with a "graph gadget" so that $F(\Gamma, \mathcal{G})$ has the same automorphisms as $C(\Gamma, \mathcal{G})$. This technique is due to Frucht $[10,11]$ and is outlined in greater detail in [2]. An example is shown in Figure 1. We call $F(\Gamma, \mathcal{G})$ the Frucht Graph of $\Gamma$ with respect to the generating set $\mathcal{G}$. The following lemma is easy to prove and also follows from the exposition in [2].

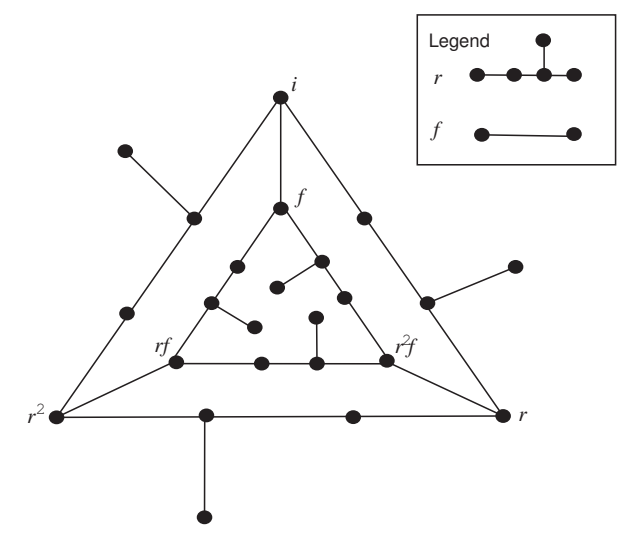

Figure 1: The Frucht graph $F\left(D_{3},\{r, f\}\right)$.

Lemma 3. For any group $\Gamma$ and any generating set $\mathcal{G}$ of $\Gamma$, $\operatorname{Aut}(C(\Gamma, \mathcal{G}))=\Gamma$ and $\operatorname{Aut}(F(\Gamma, \mathcal{G}))=\Gamma$. Furthermore, for two elements $g, h \in \Gamma$, the automorphism $g$ takes the vertex $h$ to the vertex $g h$ in both $C(\Gamma, \mathcal{G})$ and $F(\Gamma, \mathcal{G})$.

Corollary 4. If $G$ is a Cayley graph or a Frucht graph of a non-trivial group, then $\operatorname{fix}(G)=1$.

Proof. Suppose $G=F(\Gamma, \mathcal{G})$ for some group $\Gamma$ (the argument for Cayley graphs is completely analogous). Since Aut $(G)=\Gamma$ by Lemma 3 , and $\Gamma$ is not trivial by hypothesis, fix $(G)>0$. Now let $h$ be an element of $\Gamma$ (and so also a vertex in $G$ ). For any non-identity element $g \in \Gamma$, by Lemma $3, g(h)=g h \neq h$. Thus $\operatorname{stab}(h)$ is trivial, and the single-vertex set $\{h\}$ is a fixing set of $G$. 
In fact, the proof of Corollary 4 implies that every vertex of a Cayley graph is a fixing set, and every non-gadget vertex of a Frucht graph is a fixing set.

Corollary 5. For any non-trivial finite group $\Gamma, 1 \in \operatorname{fix}(\Gamma)$.

The length $l(\Gamma)$ of a finite group $\Gamma$ is the maximum number of subgroups in a chain of subgroups $e<\Gamma_{1}<\Gamma_{2}<\ldots<\Gamma_{l(\Gamma)}=\Gamma[4]$.

Proposition 6. For any finite group, $\max (\operatorname{fix}(\Gamma)) \leq l(\Gamma)$.

Proof. If $\Gamma$ is trivial, it has length 0 and fixing set $\{0\}$. Now suppose $\Gamma$ is non-trivial, and let $G$ be a graph with $\operatorname{Aut}(G)=\Gamma$. We fix a vertex $v_{1}$ in $G$ with orbit larger than one. By the Orbit-Stabilizer Theorem, $\operatorname{stab}\left(v_{1}\right)$ is a proper subgroup of $\Gamma$. If we can find a different vertex $v_{2}$ with orbit greater than one under the action of $\operatorname{stab}\left(v_{1}\right)$, we fix $v_{2}$. We continue in this way until we have fixed $G$. Since at each stage, $\operatorname{stab}\left(\left\{v_{1}, \ldots, v_{i}\right\}\right)$ is a proper subgroup of $\operatorname{stab}\left(\left\{v_{1}, \ldots, v_{i-1}\right\}\right)$, we cannot have fixed more than the length of the group.

Corollary 7. Let $k$ be the number of primes in the prime factorization of $|\Gamma|$, counting multiplicities. Then $\max (\operatorname{fix}(\Gamma)) \leq k$.

Example 8. The graph $C_{6}$ has automorphism group $D_{6}$ and fixing number 2. The graph $C_{3} \cup P_{2}$ has automorphism group $D_{6}$ and fixing number 3. On the other hand, $\left|D_{6}\right|=$ $12=2 \cdot 2 \cdot 3$. Hence fix $\left(D_{6}\right)=\{1,2,3\}$ by Corollaries 5 and $\%$.

Example 9. The graph shown in Figure 2 has automorphism group $A_{4}$ and fixing number 2. On the other hand, $\left|A_{4}\right|=12=2 \cdot 2 \cdot 3$. So $\{1,2\} \subseteq \operatorname{fix}\left(A_{4}\right) \subseteq\{1,2,3\}$, again by Corollaries 5 and 7 . Lemma 10 shows that $3 \notin \operatorname{fix}\left(A_{4}\right)$, so in fact $\operatorname{fix}\left(A_{4}\right)=\{1,2\}$.

Lemma 10. There is no graph $G$ with $\operatorname{fix}(G)=3$ and $\operatorname{Aut}(G)=A_{4}$.

Proof. Suppose by way of contradiction that $G$ is a graph with $\operatorname{fix}(G)=3$ and $\operatorname{Aut}(G)=$ $A_{4}$. Let $S=\left\{v_{1}, v_{2}, v_{3}\right\}$ be a minimum size fixing set of $G$. Note that $\operatorname{stab}\left(v_{1}\right), \operatorname{stab}\left(v_{2}\right)$, and $\operatorname{stab}\left(v_{3}\right)$ are all proper subgroups of $A_{4}$. Therefore they must be isomorphic to $\mathbb{Z}_{2}$, $\mathbb{Z}_{2} \times \mathbb{Z}_{2}$, or $\mathbb{Z}_{3}$. But if any of them have order less than 4 , fixing that vertex and one other will fix $G$, and $\operatorname{fix}(G)=2$. So $\operatorname{stab}\left(v_{1}\right) \cong \operatorname{stab}\left(v_{2}\right) \cong \operatorname{stab}\left(v_{3}\right) \cong \mathbb{Z}_{2} \times \mathbb{Z}_{2}$. But there is only one copy of $\mathbb{Z}_{2} \times \mathbb{Z}_{2}$ in $A_{4}$, $\operatorname{son} \operatorname{stab}\left(v_{1}\right)=\operatorname{stab}\left(v_{2}\right)=\operatorname{stab}\left(v_{3}\right)$, and this subgroup must therefore also equal $\operatorname{stab}\left(\left\{v_{1}, v_{2}, v_{3}\right\}\right)$. So $\left\{v_{1}, v_{2}, v_{3}\right\}$ is not a fixing set of $G$, which is a contradiction.

Lemma 11. Suppose $G$ is a graph, $\Gamma=\operatorname{Aut}(G)$ is a finite non-trivial group, and $g \in \Gamma$ is an element of order $p^{k}$, for $p$ prime and $k$ a positive integer. Then there exists a set of $p^{k}$ vertices $v_{1}, \ldots, v_{p^{k}}$ in $G$ such that, as a permutation of the vertices of $G, g$ contains the cycle $\left(v_{1} \ldots v_{p^{k}}\right)$.

Proof. Since $g$ has order $p^{k}$, the cycle decomposition of $g$ must include a cycle of length $p^{k}$. Label these vertices $v_{1}, \ldots, v_{p^{k}}$. 


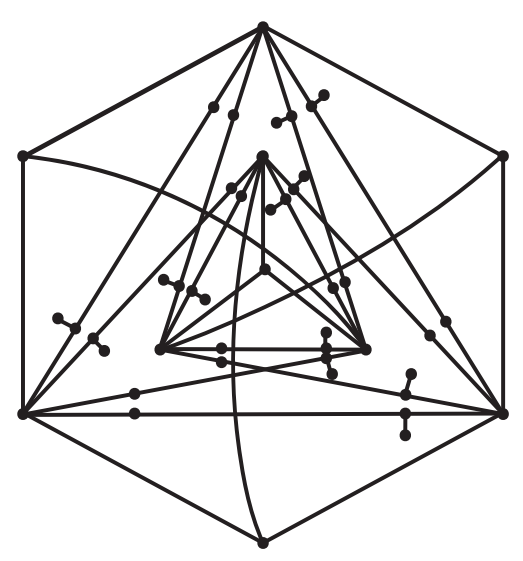

Figure 2: A graph $G$ with $\operatorname{Aut}(G)=A_{4}$ and $\operatorname{fix}(G)=2$.

Recall that the cartesian product of two groups $\Gamma_{1}$ and $\Gamma_{2}$ is the group $\Gamma_{1} \times \Gamma_{2}=$ $\left\{(g, h) \mid g \in \Gamma_{1}, h \in \Gamma_{2}\right\}$ with group operation defined by $\left(g_{1}, h_{1}\right)\left(g_{2}, h_{2}\right)=\left(g_{1} g_{2}, h_{1} h_{2}\right)$. Recall also that the sum of two sets $S$ and $T$ is $S+T=\{s+t \mid s \in S, t \in T\}$.

Lemma 12. If $\Gamma_{1}$ and $\Gamma_{2}$ are finite non-trivial groups, then $\operatorname{fix}\left(\Gamma_{1}\right)+\operatorname{fix}\left(\Gamma_{2}\right) \subseteq \operatorname{fix}\left(\Gamma_{1} \times \Gamma_{2}\right)$.

Proof. Let $a \in \operatorname{fix}\left(\Gamma_{1}\right)$ and $b \in \operatorname{fix}\left(\Gamma_{2}\right)$. Then there exist graphs $G_{1}$ and $G_{2}$ with $\operatorname{Aut}\left(G_{1}\right)=$ $\Gamma_{1}, \operatorname{Aut}\left(G_{2}\right)=\Gamma_{2}$, fix $\left(G_{1}\right)=a$, and fix $\left(G_{2}\right)=b$. Let $G_{2}^{\prime}$ be the graph obtained from $G_{2}$ by attaching the graph $Y_{k}$ shown in Figure 3 for some large value of $k$ (for example, $\left.\left|G_{1}\right|+\left|G_{2}\right|\right)$ to each vertex of $G_{2}$ at the vertex $a$. Now consider the graph $H=G_{1} \cup G_{2}^{\prime}$, the disjoint union of the graphs $G_{1}$ and $G_{2}^{\prime}$. This graph has no automorphisms that exchange vertices between $G_{1}$ and $G_{2}$, so we must have $\operatorname{Aut}(H) \cong \operatorname{Aut}\left(G_{1}\right) \times \operatorname{Aut}\left(G_{2}^{\prime}\right) \cong$ $\operatorname{Aut}\left(G_{1}\right) \times \operatorname{Aut}\left(G_{2}\right) \cong \Gamma_{1} \times \Gamma_{2}$. Furthermore, $H$ is fixed if and only if both $G_{1}$ and $G_{2}$ are fixed, so $\operatorname{fix}(H)=a+b$. Therefore $a+b \in \operatorname{fix}\left(\Gamma_{1} \times \Gamma_{2}\right)$.

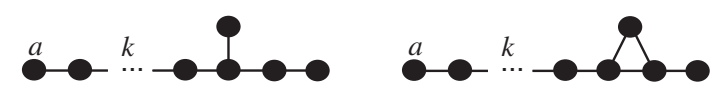

Figure 3: The graph $Y_{k}$ in the proof of Lemma 12 is shown on the left, and the graph $A_{k}$ in the proof of Theorem 14 is shown on the right.

Note that for two finite non-trivial groups $\Gamma_{1}$ and $\Gamma_{2}, 1 \in \operatorname{fix}\left(\Gamma_{1} \times \Gamma_{2}\right)$ but $1 \notin \operatorname{fix}\left(\Gamma_{1}\right)+$ $\operatorname{fix}\left(\Gamma_{2}\right)$.

Open Question. Is it true that for all finite non-trivial groups $\Gamma_{1}$ and $\Gamma_{2}$, $f(x)\left(\Gamma_{1}\right)+$ $\operatorname{fix}\left(\Gamma_{2}\right)=\operatorname{fix}\left(\Gamma_{1} \times \Gamma_{2}\right) \backslash\{1\}$ ? 


\subsection{Abelian groups}

Lemma 13. If $p$ is prime and $k$ is a positive integer, then $\operatorname{fix}\left(\mathbb{Z}_{p^{k}}\right)=\{1\}$.

Proof. By Corollary $5,1 \in$ fix $\left(\mathbb{Z}_{p^{k}}\right)$. Conversely, suppose that there exists a graph $G$ such that $\operatorname{Aut}(G)=\mathbb{Z}_{p^{k}}$. By Lemma 11, there exists a vertex in $G$ with orbit size $p^{k}$. By the Orbit-Stabilizer Theorem, fixing this vertex must fix the graph.

Let $\Gamma$ be a finite abelian group with order $n$, and let $n=p_{1}^{i_{1}} \cdots p_{k}^{i_{k}}$ be the prime factorization of $n$. Recall that there is a unique factorization $\Gamma=\Lambda_{1} \times \cdots \times \Lambda_{k}$, where $\left|\Lambda_{j}\right|=p_{j}^{i_{j}}, \Lambda_{j}=\mathbb{Z}_{p_{j}^{\alpha_{1}}} \times \cdots \times \mathbb{Z}_{p_{j}^{\alpha_{t}}}$, and $\alpha_{1}+\ldots+\alpha_{t}=i_{j}$. The numbers $p_{j}^{\alpha_{r}}$ are called the elementary divisors of $\Gamma[8]$.

Theorem 14. Let $\Gamma$ be a finite abelian group, and let $k$ be the number of elementary divisors of $\Gamma$. Then $\operatorname{fix}(\Gamma)=\{1, \ldots, k\}$.

Proof. Let $\Gamma=\Gamma_{1} \times \ldots \times \Gamma_{k}$ be the elementary divisor decomposition of $\Gamma$. For every $1 \leq i \leq k$, let $H_{i}=F\left(\Gamma_{i} \times \ldots \times \Gamma_{k}, \mathcal{G}\right)$ be any Frucht graph of $\Gamma_{i} \times \ldots \times \Gamma_{k}$. There are an infinite number of finite graphs with automorphism group $\mathbb{Z}_{n}$ and fixing number 1; for example, every graph in the family of graphs shown in Figure 4 has automorphism group $\mathbb{Z}_{5}$ and fixing number 1 . We may therefore let $G_{1}, \ldots, G_{k}$ be distinct graphs, not isomorphic to $H_{i}$ for any $i$, with automorphism groups $\Gamma_{1}, \ldots, \Gamma_{k}$, respectively, and fixing number 1. Let $G$ be the disjoint union $\left(\bigcup_{j=1}^{i-1} G_{j}\right) \cup H_{i}$. We also choose $G_{1}, \ldots, G_{k}$ so that no automorphism of $G$ moves a vertex from one $G_{j}$ to another, or from any $G_{j}$ to $H_{i}$, or vice versa. The graphs shown in Figure 4 are examples of graphs $G_{j}$ which have this property.

Then $G$ has automorphism group $\Gamma$. Furthermore, every fixing set of $G$ must include at least one vertex from each subgraph $G_{j}$ and at least one vertex from $H_{i}$, and any set with exactly one vertex moved by an automorphism from each $G_{j}$ and from $H_{i}$ is a fixing set of $G$. Therefore $\operatorname{fix}(G)=i$. Since we have constructed a graph $G$ with $\operatorname{Aut}(G)=\Gamma$ and $\operatorname{fix}(G)=i$ for any $1 \leq i \leq k,\{1, \ldots, k\} \subseteq \operatorname{fix}(\Gamma)$.

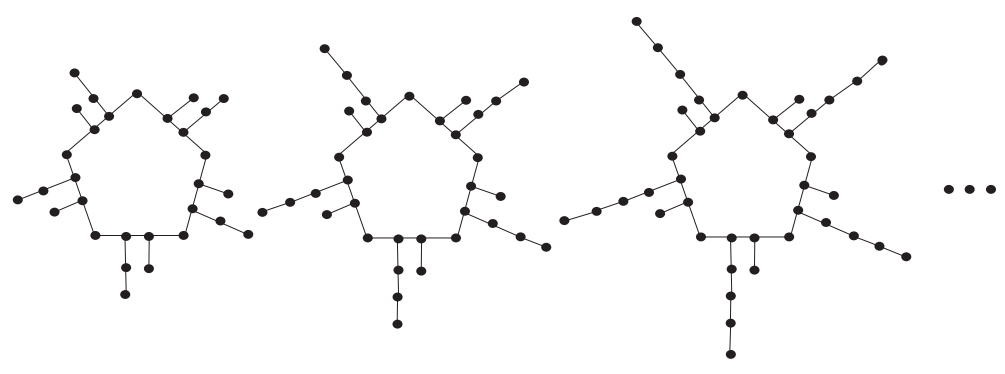

Figure 4: An infinite family of graphs with automorphism group $\mathbb{Z}_{5}$ and fixing number 1.

We prove the reverse inclusion by induction. Suppose $\Gamma$ is a finite abelian group and $G$ is a finite graph with $\operatorname{Aut}(G)=\Gamma$. If $\Gamma$ has one elementary divisor, then the result follows from Lemma 13. Suppose that $\Gamma$ has $k>1$ elementary divisors. We choose an 
elementary divisor $p^{m}$ of $\Gamma$. Then $\Gamma=\mathbb{Z}_{p^{m}} \times \Gamma^{\prime}$ for a smaller finite abelian group $\Gamma^{\prime}$. Let $g$ be a generator of the subgroup $\mathbb{Z}_{p^{m}}$ of $\Gamma$. By Lemma 11, there exists a set of $p^{m}$ vertices $v_{1}, \ldots, v_{p^{m}}$ in $G$ such that, as a permutation of the vertices of $G, g$ contains the cycle $\left(v_{1} \ldots v_{p^{m}}\right)$.

Let $H$ be the connected component of $G$ containing $v_{1}$. If $H$ is a tree, let $G^{\prime}$ be the graph obtained from $G$ by attaching the graph $A_{|G|}$ shown in Figure 3 to $G$ by identifying the vertex $a$ in $A_{|G|}$ with the vertex $v_{1}$ in $G$. Otherwise, let $G^{\prime}$ be the graph obtained from $G$ by attaching the graph $Y_{|G|}$ shown in Figure 3 to $G$ by identifying the vertex $a$ in $Y_{|G|}$ with the vertex $v_{1}$ in $G$. Denote the subgraph $A_{|G|}$ or $Y_{|G|}$ in $G^{\prime}$ by $H^{\prime}$. We claim that $\operatorname{Aut}\left(G^{\prime}\right)$ is a subgroup of $\Gamma^{\prime}$. First, we show that $G^{\prime}$ does not have any additional automorphisms that $G$ does not have. Suppose $h$ is an automorphism of $G^{\prime}$ and not $G$. So $h$ must move some vertex of $H^{\prime}$. Since $H^{\prime}$ has no automorphisms itself, $h$ must move all of its vertices. Furthermore, since $H^{\prime}$ has more vertices than $G, h$ must send a vertex of $H^{\prime}$ to another vertex of $H^{\prime}$. This means that as a permutation of the vertices of the component $H \cup H^{\prime}, h$ is completely determined: $h$ must be a flip of $H \cup H^{\prime}$ about some vertex of $H^{\prime}$. This cannot happen, since by construction $H^{\prime}$ contains a cycle if and only if $H$ does not.

Second, $v_{1}$ has larger degree in $G^{\prime}$ than in $G$, so there are no automorphisms of $G^{\prime}$ mapping $v_{1}$ to any other vertex $v_{2}, \ldots, v_{p^{m}}$. Since $g$ maps $v_{1}$ to $v_{2}, g$ does not extend to any automorphism of $G^{\prime}$.

Hence by induction $G^{\prime}$ has fixing number at most $k-1$. If $S$ is a fixing set of $G^{\prime}$ with $|S| \leq k-1$, then $S^{\prime}=S \cup\left\{v_{1}\right\}$ is a fixing set of $G$ with $\left|S^{\prime}\right| \leq k$. Therefore $G$ has fixing number at most $k$, and $\operatorname{fix}(\Gamma)=\{1, \ldots, k\}$.

\subsection{Symmetric groups}

The inflation of a graph $G, \operatorname{Inf}(G)$, is a graph with a vertex for each ordered pair $(v, e)$, where $v$ and $e$ are a vertex and an edge of $G$, and $v$ and $e$ are incident. $\operatorname{Inf}(G)$ has an edge between $\left(v_{1}, e_{1}\right)$ and $\left(v_{2}, e_{2}\right)$ if $v_{1}=v_{2}$ or $e_{1}=e_{2}$. We denote the $k$-fold inflation of the graph $G$ by $\operatorname{Inf}^{k}(G)$.

For a positive integer $n$, let $G_{k}$ be the graph with a vertex for each sequence $\left(x_{1}, \ldots\right.$, $x_{k+1}$ ) of $k+1$ integers from the set $\{1, \ldots, n\}$ with $x_{1}$ different from the remaining integers in the sequence. Vertices $u=\left(u_{1}, \ldots, u_{k+1}\right)$ and $v=\left(v_{1}, \ldots, v_{k+1}\right)$ are adjacent if and only if there exists some index $i$ such that $u_{j}=v_{j}$ for all $j<i, u_{i} \neq v_{i}$, and $u_{j}=v_{i}$ and $v_{j}=u_{i}$ for all $j>i$.

Lemma 15. The graphs $G_{k}$ and $\operatorname{Inf}^{k}\left(K_{n}\right)$ are isomorphic.

Proof. We define an isomorphism $\varphi: \operatorname{Inf}^{k}\left(K_{n}\right) \rightarrow G_{k}$ inductively. For the base case, note that $\operatorname{Inf}^{0}\left(K_{n}\right) \cong G_{0} \cong K_{n}$. Now assume $\varphi^{\prime}: \operatorname{Inf}^{k-1}\left(K_{n}\right) \rightarrow G_{k-1}$ is an isomorphism, and suppose that $v$ is a vertex in $\operatorname{Inf}^{k}\left(K_{n}\right)$. By the definition of the inflation, $v=\left(v^{\prime}, e^{\prime}\right)$, where $v^{\prime}$ is a vertex in $\operatorname{Inf}^{k-1}\left(K_{n}\right)$ and $e^{\prime}$ is an edge in $\operatorname{Inf}^{k-1}\left(K_{n}\right)$. So $\varphi^{\prime}\left(v^{\prime}\right)=\left(a_{1}, \ldots, a_{k}\right)$ and $e^{\prime}=\left\{v^{\prime}, u^{\prime}\right\}$ where $\varphi^{\prime}\left(u^{\prime}\right)=\left(b_{1}, \ldots, b_{k}\right)$, for two vertices $\left(a_{1}, \ldots, a_{k}\right)$ and $\left(b_{1}, \ldots, b_{k}\right)$ in $G_{k-1}$. Since $v^{\prime} \sim u^{\prime}$, by the definition of $G_{k-1}$, there exists an index $1 \leq i \leq k$ such 

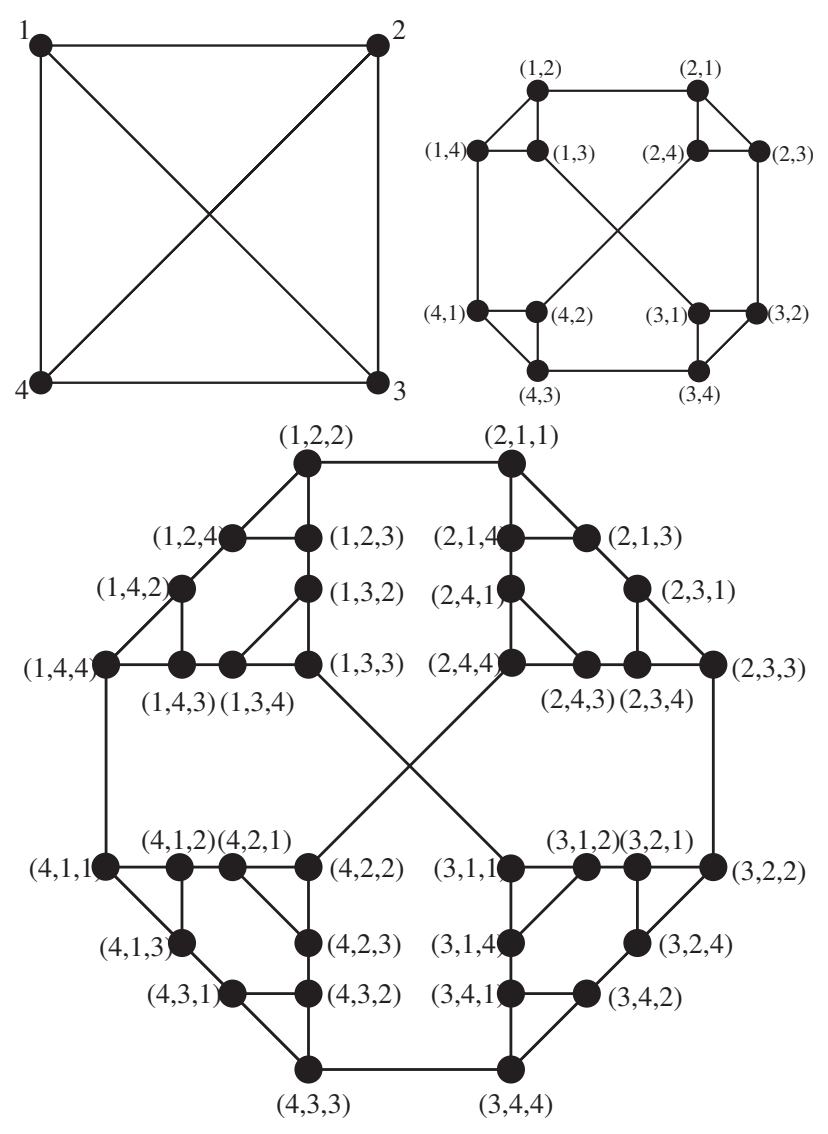

Figure 5: The graph $K_{4}$ and its first and second inflations.

that $a_{j}=b_{j}$ for all $1 \leq j<i, a_{i} \neq b_{i}$, and $a_{j}=b_{i}$ and $b_{j}=a_{i}$ for all $i<j \leq k$. We define $\varphi(v)=\left(a_{1}, \ldots, a_{k}, b_{i}\right)$. Note that since $\varphi^{\prime}$ is a bijection by induction, it is easy to see that $\varphi$ is a bijection as well.

We now prove that $\varphi$ is an isomorphism. First suppose that $v$ and $u$ are adjacent vertices of $\operatorname{Inf}^{k}\left(K_{n}\right)$. By the definition of inflation, $v=\left(v^{\prime}, e^{\prime}\right)$ and $u=\left(u^{\prime}, d^{\prime}\right)$ for two vertices $v^{\prime}$ and $u^{\prime}$ in $\operatorname{Inf}^{k-1}\left(K_{n}\right)$ and two edges $e^{\prime}$ and $d^{\prime}$ in $\operatorname{Inf}^{k-1}\left(K_{n}\right)$ incident to $v^{\prime}$ and $u^{\prime}$, respectively. By the definition of adjacency in $\operatorname{Inf}^{k}\left(K_{n}\right)$, either $v^{\prime}=u^{\prime}$ or $e^{\prime}=d^{\prime}$.

Case 1. $v^{\prime}=u^{\prime}$. In this case, $\varphi^{\prime}\left(v^{\prime}\right)=\varphi^{\prime}\left(u^{\prime}\right)=\left(a_{1}, \ldots, a_{k}\right)$, so $\varphi(v)$ and $\varphi(u)$ differ only in their last coordinate. Therefore $\varphi(v) \sim \varphi(u)$ by the definition of adjacency in $G_{k}$.

Case 2. $e^{\prime}=d^{\prime}$. Since $e^{\prime}$ is incident to $v^{\prime}$ and $d^{\prime}$ is incident to $u^{\prime}, e^{\prime}=d^{\prime}$ must be the edge between the vertices $v^{\prime}$ and $u^{\prime}$. So $\varphi^{\prime}\left(v^{\prime}\right) \sim \varphi^{\prime}\left(u^{\prime}\right)$, hence $\varphi^{\prime}\left(v^{\prime}\right)$ and $\varphi^{\prime}\left(u^{\prime}\right)$ must satisfy the definition of adjacency in $G_{k-1}$. By the definition of $\varphi, \varphi(v)$ and $\varphi(u)$ are still adjacent in $G_{k}$.

Now suppose that $v$ and $u$ are non-adjacent vertices of $\operatorname{Inf}^{k}\left(K_{n}\right)$, and again let $v=$ $\left(v^{\prime}, e^{\prime}\right)$ and $u=\left(u^{\prime}, d^{\prime}\right)$. By the definition of adjacency in $\operatorname{Inf}^{k}\left(K_{n}\right), v^{\prime} \neq u^{\prime}$ and $e^{\prime} \neq d^{\prime}$.

Case 1. $v^{\prime}$ is not adjacent to $u^{\prime}$. So $\varphi^{\prime}\left(v^{\prime}\right) \not \varphi^{\prime}\left(u^{\prime}\right)$, so the sequences $\varphi^{\prime}\left(v^{\prime}\right)$ and $\varphi^{\prime}\left(u^{\prime}\right)$ do not satisfy the definition of adjacency in $G_{k-1}$. Since $\varphi(v)$ and $\varphi(u)$ are formed from 
$\varphi^{\prime}\left(v^{\prime}\right)$ and $\varphi^{\prime}\left(u^{\prime}\right)$ by appending an extra number to their sequences, the new sequences $\varphi(v)$ and $\varphi(u)$ still do not satisfy the definition of adjacency in $G_{k}$.

Case 2. $v^{\prime}$ is adjacent to $u^{\prime}$. Since $v^{\prime} \neq u^{\prime}, \varphi^{\prime}\left(v^{\prime}\right)$ and $\varphi^{\prime}\left(u^{\prime}\right)$ differ in their $k$ th coordinate. But since $e^{\prime} \neq d^{\prime}$, either the $(k+1)$ st coordinate of $\varphi(v)$ differs from the $k$ th coordinate of $\varphi(v)$, or the $(k+1)$ st coordinate of $\varphi(u)$ differs from the $k$ th coordinate of $\varphi(u)$. Therefore $\varphi(v)$ is not adjacent to $\varphi(u)$ in $G_{k}$.

By Lemma 15, we may label the vertices of $\operatorname{Inf}^{k}\left(K_{n}\right)$ using the vertices of $G_{k}$, and follow the rule for adjacency of vertices in $\operatorname{Inf}^{k}\left(K_{n}\right)$ given by the definition of $G_{k}$. We do this for the remainder of this section.

Theorem 16. For $n>3$ and $k \geq 0, \operatorname{Aut}\left(\operatorname{Inf}^{k}\left(K_{n}\right)\right)=S_{n}$ and $\operatorname{fix}\left(\operatorname{Inf}^{k}\left(K_{n}\right)\right)=\left\lceil\frac{n-1}{k+1}\right\rceil$.

Proof. The statement is clear for $k=0$, so assume $k>0$. Since each vertex of $\operatorname{Inf}^{k}\left(K_{n}\right)$ is labeled with a sequence of the numbers $\{1, \ldots, n\}$ of length $k+1$ by Lemma 15 , every permutation $g$ in $S_{n}$ induces a natural permutation of the vertices of $\operatorname{Inf}^{k}\left(K_{n}\right)$. Again by Lemma 15, it is easy to see that these permutations are all automorphisms of $\operatorname{Inf}^{k}\left(K_{n}\right)$. So $S_{n}<\operatorname{Aut}\left(\operatorname{Inf}^{k}\left(K_{n}\right)\right)$.

Now suppose that $g \in \operatorname{Aut}\left(\operatorname{Inf}^{k}\left(K_{n}\right)\right)$. We show that $g$ is determined as a permutation of the numbers 1 through $n$ in the labeling sequences of the vertices of $\operatorname{Inf}^{k}\left(K_{n}\right)$, and therefore $g \in S_{n}$. Suppose $v=\left(a_{1}, \ldots, a_{k+1}\right)$ and $w=\left(b_{1}, \ldots, b_{k+1}\right)$ are two vertices in $\operatorname{Inf}^{k}\left(K_{n}\right)$. By the definition of adjacency in $G_{k}$, if $a_{i}=b_{i}$ for $1 \leq i \leq k$, then $v$ and $w$ are adjacent. Therefore if we partition $\operatorname{Inf}^{k}\left(K_{n}\right)$ into blocks of vertices with the same first $k$ elements in their labeling sequence, each block forms a maximal clique of $\operatorname{Inf}^{k}\left(K_{n}\right)$. The graph formed by contracting each of these maximal cliques to a single vertex is $\operatorname{Inf}^{k-1}\left(K_{n}\right)$. Since maximal cliques are preserved under automorphisms, the automorphism $g$ induces a natural automorphism $g^{\prime}$ on $\operatorname{Inf}^{k-1}\left(K_{n}\right)$. By induction, $g^{\prime}$ is determined as a permutation $p$ of the numbers 1 through $n$ in the labeling sequences of the vertices of $\operatorname{Inf}^{k-1}\left(K_{n}\right)$. Now $g$ is determined by the same permutation $p$, since the action of $p$ on $\left(a_{1}, \ldots, a_{k}\right)$ determines which maximal clique contains $g(v)$, and the action of $p$ on $a_{k+1}$ determines $g(v)$ within that maximal clique.

By the definition of the correspondence between an element $g$ of $\operatorname{Aut}\left(\operatorname{Inf}^{k}\left(K_{n}\right)\right)$ and its corresponding permutation $p$ in $S_{n}$, for any vertex $v=\left(a_{1}, \ldots, a_{k+1}\right)$ of $\operatorname{Inf}^{k}\left(K_{n}\right), g(v)=v$ if and only if $p\left(a_{i}\right)=a_{i}$ for all $1 \leq i \leq k+1$. Therefore $\operatorname{stab}(v)=\operatorname{stab}\left(\left\{a_{1}, \ldots, a_{k+1}\right\}\right)$. This means that any set of vertices whose vertex labels include the set $\{1, \ldots, n-1\}$ is a fixing set of $\operatorname{Inf}^{k}\left(K_{n}\right)$. One such set is $\{(1, \ldots, k+1),(k+2, \ldots, 2 k+1), \ldots,(m k+$ $m+1, \ldots, m k+m+k+1),(n-k-1, \ldots, n-1)\}$, where $m=\left\lfloor\frac{n-1}{k+1}\right\rfloor$. This set has $\left\lceil\frac{n-1}{k+1}\right\rceil$ vertices. Conversely, any set $S$ of vertices whose vertex labels do not include any two of the numbers 1 through $n$, say $i$ and $j$, cannot be a fixing set, since the element of $\operatorname{Aut}\left(\operatorname{Inf}^{k}\left(K_{n}\right)\right)$ corresponding to the transposition $(i, j)$ is a non-identity element of the stabilizer of $S$. This clearly requires at least $\left\lceil\frac{n-1}{k+1}\right\rceil$ vertices, so $\operatorname{fix}\left(\operatorname{Inf}^{k}\left(K_{n}\right)\right)=\left\lceil\frac{n-1}{k+1}\right\rceil$.

It seems likely that the proof of Theorem 16 could extend to inflations of graphs other than $K_{n}$. However, since $\operatorname{Inf}^{k}\left(C_{n}\right)=C_{2^{k} n}$, fix $\left(\operatorname{Inf}^{k}\left(C_{n}\right)\right)=2$ for all $k \geq 0$ and $n \geq 3$. This motivates the following question. 
Open Question. For which graphs $G$ is it true that $\operatorname{fix}\left(\operatorname{Inf}^{k}(G)\right)=\left\lceil\frac{\operatorname{fix}(G)}{k+1}\right\rceil$ ?

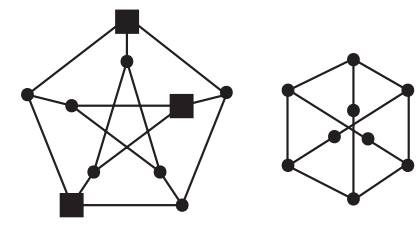

Figure 6: The Petersen graph with a fixing set shown as square vertices, and the Petersen graph with one vertex deleted.

Proposition 17. The Petersen graph $P$ has automorphism group $S_{5}$ and fixing number 3.

Proof. Many proofs that $\operatorname{Aut}(P)=S_{5}$ appear in the literature; one can be found in [2]. A fixing set of $P$ with 3 vertices is shown in Figure 6. It remains to show that any fixing set of $P$ has at least 3 vertices. Suppose that $S=\left\{v_{1}, \ldots, v_{k}\right\}$ is a fixing set of $P$. Since $P$ is vertex-transitive [2], we may choose $v_{1}$ to be any vertex of $P$. Since automorphisms in $\operatorname{stab}\left(v_{1}\right)$ preserve distance from $v_{1}$, any element of $\operatorname{stab}\left(v_{1}\right)$ must permute the three vertices adjacent to $v_{1}$ among themselves, and the six vertices that are distance two from $v_{1}$ among themselves. Since automorphisms of $P-v_{1}$ also have this property, fixing the rest of $P$ is equivalent to fixing the graph $P-v_{1}$. This graph is shown in Figure 6 , and has fixing number 2 since its automorphisms are the same as the automorphisms of $C_{6}$.

Lemma 18. For any positive integer $n$, if $i$ is a prime power dividing $n$ !, and $j$ is the number of prime factors of $n ! / i$, counting multiplicities, then $\max \left(\operatorname{fix}\left(S_{n}\right)\right) \leq j+1$.

Proof. Let $G$ be a graph with $\operatorname{Aut}(G)=S_{n}$. Let $g$ be an element of $S_{n}$ with order $i$. Since $i$ is a prime power, by Lemma 11, as a permutation of the vertices of $G, g$ contains a cycle of order $i$. Let $v$ be a vertex in this cycle, and fix $v$. Since $g$ is not an element of $\operatorname{stab}(v)$, $|\operatorname{stab}(v)| \leq n ! / i$. Hence $G$ can be fixed with $j$ additional vertices by Lemma 7 .

We conjecture that this lemma can be improved by fixing more than one vertex. However, one cannot use induction since the group $\operatorname{stab}(v)$ in the proof of Lemma 18 may not be symmetric.

We also have an upper bound on $\max \left(\operatorname{fix}\left(S_{n}\right)\right)$ given by the following lemma, which appears in [4].

Lemma 19. $l\left(S_{n}\right)=\lceil 3 n / 2\rceil-b(n)-1$, where $b(n)$ is the number of ones in the binary representation of $n$.

The following table gives lower and upper bounds on the set fix $\left(S_{n}\right)$, given by Propositions $6,16,17,18$, and 19. Note that Lemma 18 is the better upper bound for $n \leq 8$, and Lemmas 6 and 19 are better for $n \geq 10$. 


\begin{tabular}{|c|c|c|}
\hline group & lower bound & upper bound \\
\hline$S_{2}$ & $\{1\}$ & $\{1\}$ \\
$S_{3}$ & $\{1,2\}$ & $\{1,2\}$ \\
$S_{4}$ & $\{1,2,3\}$ & $\{1,2,3\}$ \\
$S_{5}$ & $\{1,2,3,4\}$ & $\{1,2,3,4\}$ \\
$S_{6}$ & $\{1,2,3,5\}$ & $\{1,2,3,4,5,6\}$ \\
$S_{7}$ & $\{1,2,3,6\}$ & $\{1,2,3,4,5,6,7\}$ \\
$S_{8}$ & $\{1,2,3,4,7\}$ & $\{1,2,3,4,5,6,7,8,9\}$ \\
$S_{9}$ & $\{1,2,3,4,8\}$ & $\{1,2,3,4,5,6,7,8,9,10,11\}$ \\
$S_{10}$ & $\{1,2,3,5,9\}$ & $\{1,2,3,4,5,6,7,8,9,10,11,12\}$ \\
\hline
\end{tabular}

Motivated by the first four rows of the table, we make the following conjecture.

Conjecture 20. $\operatorname{fix}\left(S_{n}\right)=\{1, \ldots, n-1\}$.

Of particular interest is the potential gap which occurs first in $\operatorname{fix}\left(S_{6}\right)$. More generally, all known examples of fixing sets of non-trivial finite groups are of the form $\{1, \ldots, k\}$ for some $k$. If the fixing set of every non-trivial finite group is of this form, then the computation of a fixing set becomes much easier: we need only to find the largest value in the set, which we may then call the fixing number of the group.

Open Question. For every non-trivial finite group $\Gamma$, does there exist a positive integer $k$ such that $\operatorname{fix}(\Gamma)=\{1, \ldots, k\}$ ?

\section{Acknowledgements}

We thank Pete L. Clark and an anonymous reviewer for many helpful suggestions.

\section{References}

[1] Michael O. Albertson and Karen L. Collins. Symmetry breaking in graphs. Electron. J. Combin., 3(1):Research Paper 18, approx. 17 pp. (electronic), 1996.

[2] Lowell W. Beineke and Robin J. Wilson, editors. Graph connections: Relationships between graph theory and other areas of mathematics, volume 5 of Oxford Lecture Series in Mathematics and its Applications. The Clarendon Press Oxford University Press, New York, 1997.

[3] Debra Boutin. Identifying graph automorphisms using determining sets. Electron. J. Combin., 13(1):Research Paper 78, approx. 14 pp. (electronic), 2006.

[4] Peter J. Cameron, Ron Solomon, and Alexandre Turull. Chains of subgroups in symmetric groups. J. Algebra, 127(2):340-352, 1989.

[5] Karen Collins and Joshua D. Laison. Fixing numbers of Kneser graphs. preprint, 2008. 
[6] Karen L. Collins and Ann N. Trenk. The distinguishing chromatic number. Electron. J. Combin., 13(1):Research Paper 16, 19 pp. (electronic), 2006.

[7] John D. Dixon and Brian Mortimer. Permutation groups, volume 163 of Graduate Texts in Mathematics. Springer-Verlag, New York, 1996.

[8] David S. Dummit and Richard M. Foote. Abstract algebra. John Wiley and Sons, Inc., Hoboken, NJ, 3 edition, 2004.

[9] David Erwin and Frank Harary. Destroying automorphisms by fixing nodes. Discrete Math., 306(24):3244-3252, 2006.

[10] Robert Frucht. Hertellung von graphen mit vorgegebenen abstrakten gruppen. Compositio Math., 6:239-250, 1938.

[11] Robert Frucht. Graphs of degree three with a given abstract group. Canadian J. Math., 1:365-378, 1949.

[12] Chris Godsil and Gordon Royle. Algebraic graph theory, volume 207 of Graduate Texts in Mathematics. Springer-Verlag, New York, 2001.

[13] Julianna Tymoczko. Distinguishing numbers for graphs and groups. Electron. J. Combin., 11(1):Research Paper 63, 13 pp. (electronic), 2004. 